\title{
Coronavirus Disease 2019 (COVID-19): Prevention and Control in Gynecological Outpatient Clinic
}

\author{
Dongmei Yao ${ }^{1}$, Kun Yan ${ }^{2}$, Jie Duan ${ }^{1}$, Xian Zhang ${ }^{1}$ and Limin Zhou ${ }^{1 *}$ \\ ${ }^{1}$ Department of Gynecology, Maternal and Child Health Hospital of Hubei Province, Tongji Medical College, Huazhong \\ University of Science and Technology, Wuhan, China, ${ }^{2}$ State Key Laboratory of Virology, Modern Virology Research Center, \\ College of Life Sciences, Wuhan University, Wuhan, China
}

\section{OPEN ACCESS \\ Edited by: \\ Henri Heussen,}

Cosanta BV, Netherlands

Reviewed by:

Andrea Spinazzè,

University of Insubria, Italy

John Wah Lim,

National University of

Singapore, Singapore

*Correspondence:

Limin Zhou

zhoulimin@hbfy.com

Specialty section:

This article was submitted to Occupational Health and Safety,

a section of the journal

Frontiers in Public Health

Received: 26 October 2020 Accepted: 03 December 2020 Published: 08 January 2021

Citation:

Yao D, Yan K, Duan J, Zhang X and Zhou L (2021) Coronavirus Disease 2019 (COVID-19): Prevention and Control in Gynecological Outpatient Clinic. Front. Public Health 8:618494. doi: 10.3389/fpubh.2020.618494
Objective: The pandemic of coronavirus disease 2019 (COVID-19) has become a major public health challenge around the world, and outbreaks of the SARS-CoV-2 have constituted a public health emergency of international concern. Infection control measures are necessary to prevent further spread of the virus and to help control the epidemic situation. Due to the characteristics of gynecological settings, the risk of cross infection between patients and gynecologic practitioners can be high, strict and effective infection control protocols are urgently needed. This article, based on our experience and relevant guidelines and research, introduces prevention and control measures for use in gynecological outpatient clinics and provides recommended management for gynecologists in (potentially) affected areas.

Keywords: infection control, transmission, COVID-19, gynecological public health, gynecological practice management

\section{INTRODUCTION}

On 31 December 2019, the cases of pneumonia of unknown etiology were firstly reported in Wuhan City. The number of people infected with the novel coronavirus subsequently named as SARS-CoV-2 dramatically increased worldwide, and the virus causing coronavirus disease (COVID-19) was characterized as a pandemic $(1,2)$. SARS-CoV-2 can be transmitted among humans through droplets and contacts (2-4). There is also the possibility of aerosol transmission and fecal-oral transmission (5). Patients with mild symptoms exhibit fever, dry cough and fatigue. Severe patients may have dyspnea, respiratory failure and severe infection. Our hospital is located in Wuhan, the most serious epidemic area in China. Total confirmed cases of COVID-19 were over 50,000 in Wuhan, it accounted for more than half of the total number of confirmed cases of China. However, there are only eight confirmed cases in Wuhan on 25 November 2020 (Data sources: WHO, CDC and local media reports). With improvement of the domestic epidemic situation, outpatient services are gradually recovering, but the global epidemic status is not encouraging. However, during the epidemic, there were still patients with gynecological problems requiring outpatient treatment. Gynecological patients required gynecological examinations, and some of them needed further procedures. Although no evidence of the SARS-CoV-2 can be found in the vagina, SARS-CoV-2 viral nucleic acids can be detected in the blood $(6,7)$. Moreover, healthcare workers are at risk of contracting respiratory virus infections when delivering routine care for patients infected with the viruses, and they are at risk of disseminating virus because they touch virus-contaminated fomites (810). Gynecological healthcare workers have close contact with patients, and the risks should not be underestimated. To prevent contagion during the epidemic, our gynecology clinic 
has formulated prevention and control measures and workflows during the epidemic. From January 23 to May 23, 2020, 27,212 patients were treated, 3,357 patients and their families were screened, and 14 cases were abnormal (positive for nucleic acid or SARS-CoV-2 antibody IgM). There was no infection among the healthcare workers of the gynecology clinic. The results are summarized as follows.

\section{PREVENTION AND CONTROL MEASURES IN OUTPATIENTS}

\section{Patient Management}

The outpatient service adopted an online appointment system. Patients with appointment information and their family members (only 1 person) were able to see a doctor. Patients and their families were asked to wear masks, and correct mask wearing was emphasized (11). The entrance of the hospital was equipped with an infrared temperature detector. The staff at the entrance used a hand-held temperature gun to measure the temperature of the people who entered the hospital. If the body temperature was higher than normal, they were admitted to the fever clinic. Individuals with normal body temperature could enter. Before entering the gynecological area, the triage nurse checked the health codes of the patients and their families. Based on the mobile big data provided by government, key persons, subjected to epidemic prevention and control, are indicated with red code. Persons at risk of potential epidemic are with yellow code. Other persons are indicated with green code. The patients with a green code could see a doctor, and the patients with a red code were advised to go to designated hospitals. The temperature was measured again. Then, the triage nurse marked the normal temperature and green code symbol on the treatment sheet. The patients filled out the outpatient screening form "outpatient 14 questions" (Table 1) at the triage table. Items 1-4 were epidemiological history screening, items 5-7 were clinical symptom screening, and items 8-14 were related to high risk factor screening. The patients were asked if they had high risk factors, fever, cough and other acute respiratory illness symptoms. The patients were required to fill out the form and sign the notification before entering the diagnosis room. In the diagnosis room, the doctor reconfirmed the temperature and questionnaire and then began the outpatient clinic work. Gynecological patients are usually accompanied by family members. Except for patients in emergency departments, only patients were allowed to enter the waiting area, and accompanying individuals were required to wait outside the waiting area. The safety distance between personnel was required to be more than 1.5 meters, and we marked $1.5 \mathrm{~m}$ intervals on the floor (12). There are subsequent phone contacts with patients and their family after leaving the hospital at 7th and 14th day, to confirm whether they have been diagnosed as positive for COVID-19. If a case of COVID-19 is confirmed, the clinical staff who has contacted the COVID-19 patient, as well as the patients who have contacted the staff during that period, will be tracked according to the records in the hospital's computer system. These persons at potential epidemic risk will subject to viral nucleic acid test and put in quarantine for 14 days (Table 2).

\section{Management of Gynecological Healthcare Workers}

The temperatures of gynecological healthcare workers were measured before work and any abnormal results were reported

TABLE 1 | Epidemiological investigation of COVID-19.

\begin{tabular}{|c|c|}
\hline 1 & Have you lived in a community with reported cases within 14 days? \\
\hline 2 & Have you had contact with a covid-19 infected person (nucleic acid test positive) within 14 days? \\
\hline 3 & Have you been exposed to someone with fever or respiratory symptoms within 14 days? \\
\hline 4 & Do you have 2 or more confirmed covid-19 cases or asymptomatic infections around you? \\
\hline 5 & Have you had a fever in 14 days? \\
\hline 6 & Do you have cough, expectoration, suffocation, sore throat, chest pain and other respiratory symptoms within 14 days? \\
\hline 7 & Do you have fatigue, myalgia or diarrhea within 14 days? \\
\hline 8 & Have you ever taken a plane, train, coach or been to a designated hospital within 14 days? \\
\hline 9 & Have you ever attended a party with more than 2 people or been to a public place with more than 2 people in 14 days? \\
\hline 10 & $\begin{array}{l}\text { Are you a front-line anti epidemic personnel (front-line medical staff in designated or shelter hospitals, staff involved in epidemic disposal and exposed to } \\
\text { confirmed or suspected cases)? }\end{array}$ \\
\hline 11 & $\begin{array}{l}\text { If they are frontline anti epidemic personnel, have they met the following three conditions after finishing their work (isolation for } 14 \text { days, no abnormality in } \\
\text { CT Reexamination, and negative nucleic acid test)? }\end{array}$ \\
\hline 12 & Have you ever been a confirmed or suspected patient? \\
\hline 13 & $\begin{array}{l}\text { If you have ever been a confirmed or suspected patient, did you meet the following four conditions (cured and isolated at the isolation point for } 14 \text { days, } \\
\text { and then isolated at home for } 14 \text { days after returning home, no abnormality in CT Reexamination and negative nucleic acid test)? }\end{array}$ \\
\hline 14 & Is there any other special situation or additional explanation needed? If yes, please inform the medical staff. \\
\hline & Signature: \\
\hline
\end{tabular}

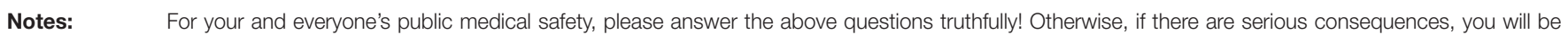
investigated for legal responsibility according to the law of the people's Republic of China on the prevention and control of infectious diseases and other relevant laws. If necessary, it will be handed over to the judicial organ for filing and handling the crime of endangering public security by dangerous means. I have answered the above questions truthfully, without any concealment. If there is any concealment, I will bear the corresponding legal responsibility! 
TABLE 2 | Track condition of patients work flow.

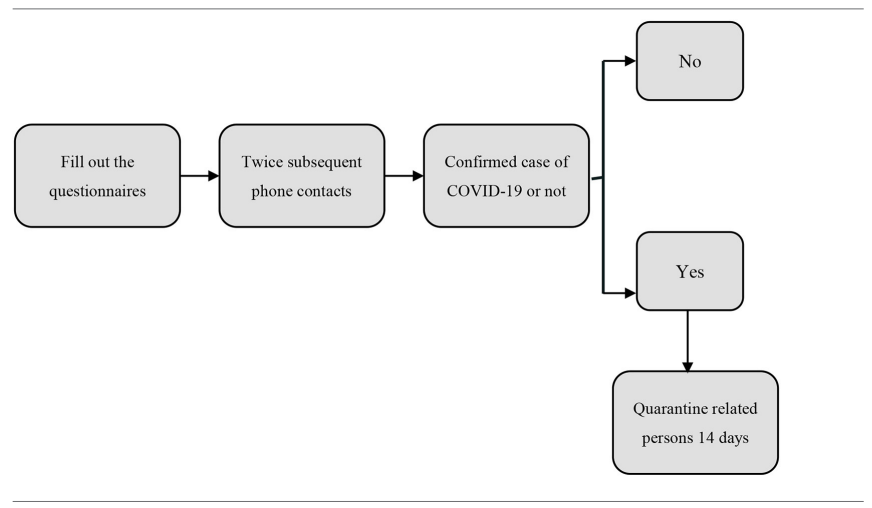

immediately. It's necessary to ensure complete hand hygiene, physical distancing and use other personal protective equipment (PPE), including masks, gloves, gowns, cap, shoe cover, and goggles or face shield, to prevent dissemination of virus. Triage nurses and doctors in the gynecology outpatient department adopted measures of no less than two-level protection. In gynecological procedure areas, such as colposcopy rooms, gynecological treatment rooms, hysteroscopy rooms, family planning procedure rooms, etc., the staff wore three-level protection, including isolation clothes, masks, gloves and shoe covers, goggles and face screens. Hand hygiene and proper mask wearing were emphasized to reduce the risk of SARS-CoV-2 pneumonia among healthcare workers. During lunch and rest time, healthcare workers were required to maintain an effective distance of more than 1.5 meters. The indoor ventilation was maintained in good condition, and staff were scheduled to eat in batches to avoid large gatherings. In addition, triage nurses and doctors accepted viral nucleic acid and antibody tests irregularly. Once a local positive case is confirmed, all clinical staff have to be screened.

\section{Site and Equipment Management}

To decrease the risk of nosocomial infection, patients could be treated in an isolated and well-ventilated room (Figure 1). In addition, central air conditioning was stopped in the waiting area, diagnosis room and outpatient operating rooms, and ventilation was strengthened (13-15). To allow for natural ventilation, windows and doors may remain open for $30 \mathrm{~min}$, at least twice a day. Besides, it is also able to control and guarantee airflow by installing a fresh air system. According to our country's technical standards, the minimum ventilation rate of the biosafety laboratory is 12 times/hour and the minimum ventilation rate of the negative pressure isolation ward is 8 times/hour. Supplement general ventilation with air disinfector and germicidal ultraviolet lights. If a patient suspected of infection was found in the clinic, for example, if a positive test for SARS-Cov-2, viral pneumonia antibody IgM, or typical CT changes were detected, the clinic room was changed immediately and disinfected with ozone. After lunch break and after work each day, an ozone disinfector was used to disinfect the diagnosis room and the operating rooms of the outpatient department. In the outpatient waiting area, a $3 \%$ hydrogen peroxide spray was used to disinfect the air. Object surfaces were wiped with $1,000 \mathrm{mg} / \mathrm{L}$ chlorine containing disinfectant or disinfected by ultraviolet radiation. It was reported that SARS-CoV-2 has been detected in anal swabs $(16,17)$, although no SARS-CoV-2 has been found in the vagina. However, in gynecological examination, the female reproductive system contacts the pad sheet directly and may contaminate the examination table. Therefore, attention is needed during gynecological examination in particular to avoid cross infection. The patients were examined with disposable pads, one each, and sodium hypochlorite (effective chlorine content: $1,000 \mathrm{mg} / \mathrm{L}$ ) were used to wipe and disinfect the examination bed and operating bed directly in contact with patients. A rapid hand spray (alcohol-based hand rub) was placed in the guide table and diagnostic room for disinfection at any time.

\section{CHARACTERISTICS OF GYNECOLOGICAL CLINICS DURING THE EPIDEMIC PERIOD}

\section{Outpatients and Disease Classification From January 23, 2020 to May 23, 2020}

A total of 27,212 patients were treated in the gynecology department (Figure 2). Before Wuhan was unsealed on April 8,2020 , most of the patients were diagnosed with pregnancyrelated conditions, including threatened abortion, missed abortion, early pregnancy (termination of pregnancy), and ectopic pregnancy, which accounted for more than $95 \%$ of the total number of outpatients. Gynecological emergencies include abnormal uterine bleeding, rupture of the corpus luteum, rupture of ectopic pregnancy, inevitable abortion and massive hemorrhage.

\section{Screening Work Flow}

Patients who needed to be admitted to the hospital or to undergo outpatient surgery [family planning surgery, outpatient cervical endoscopy, laser surgery, loop electrosurgical excision procedure (LEEP) surgery, tubal hydrotubation] were required to undergo the following examinations (Table 3): CT lung screening, routine blood tests and CRP, SARS-CoV-2 nucleic acid and SARS-CoV-2 pneumonia antibody. If typical ground glass changes were observed on $\mathrm{CT}$, the patient must go to the fever clinic; if the nucleic acid or SARS-CoV-2 antibody IgM test is positive, the patient was isolated immediately and the result reported to the medical department. If only the antibody IgG test was positive, the patient was advised to take another nucleic acid test. If the patient is in an emergency situation and needed to be hospitalized immediately, the patient was admitted to the buffer room of the ward for treatment. If necessary, only one fixed person was permitted to accompany the patient, and this person was required to undergo CT, routine blood tests, CRP, SARS-CoV-2 nucleic acid and SARS-CoV2 antibody tests. If the above results were normal, they were permitted to enter the ward to accompany the patient. We screened 3,357 cases, 14 of which had abnormal results (including 
A

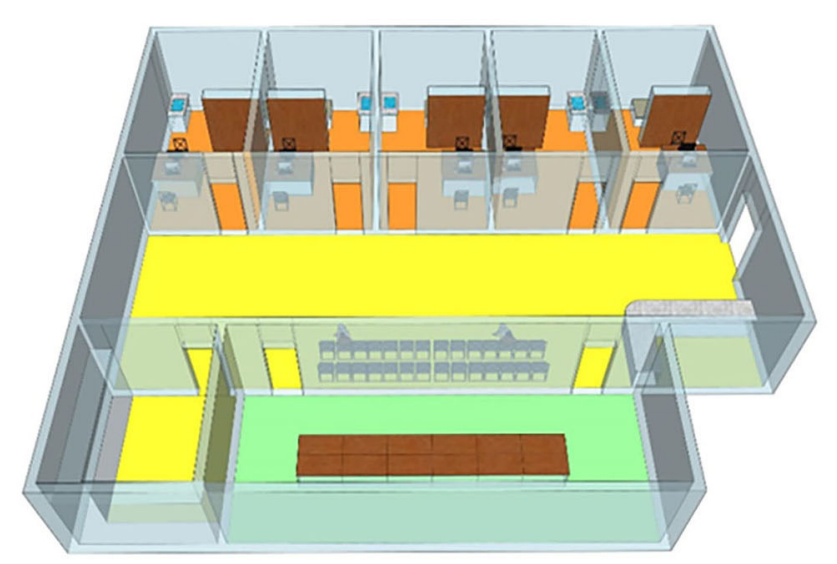

B

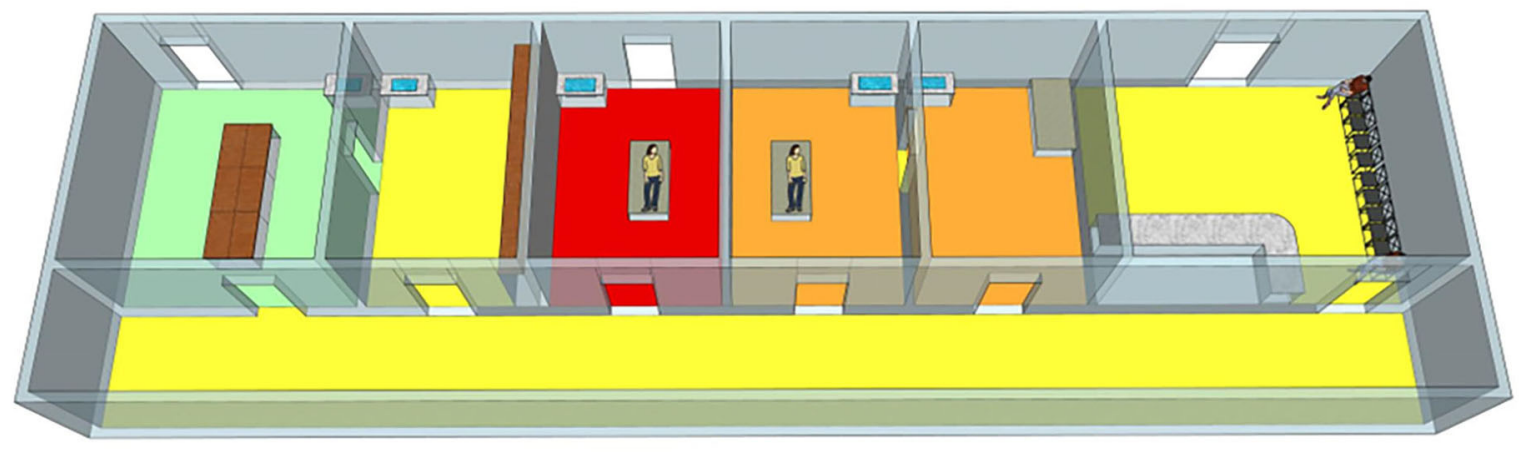

FIGURE 1 | The outpatient district (A) and operation room (B). Yellow: triage and waiting area. Orange: Gynecology clinic and operation room. Red: isolation operation room. Green: resting area for staff only. As shown in the diagram, our triage staff in the yellow area wear disposable surgical mask, cap, and work clothes. In the orange area, gynecologic staff is provided with PPE (personal protective equipment), including disposable N95 masks, gloves, gowns, cap, shoe cover, and goggles or face shield. The area is disinfected once every half day. All the patients were treated in this area. The isolation clinic in the red area is designed for patients who are suspected with COVID-19 or who are recovering from COVID-19. Separate entrances for patients. gynecologic staff should wear protective clothing besides the aforementioned PPE. In addition, the entire isolation area is disinfected immediately after the treatment is over and the patient has left. Staff can have a rest in the room (green area). They are recommended to enter the room by turn and to keep wearing medical masks unless they are eating or drinking.

positive tests for SARS-CoV-2 nucleic acid or SARS-CoV-2 antibody IgM). They were transferred to designated hospitals for further examination.

\section{Characteristics of Outpatient Operation}

All specimens that may be considered potential risks for biological contamination, such as leucorrhea specimen test tubes, cervical cytology specimen bottles, and pathological examination specimens, were placed in specimen bags for inspection. The outpatient family planning operating room was divided to provide a special area for patients in need of emergency treatment with no time for screening. In strict compliance with the above procedures for prevention and control and screening, none of the healthcare workers in the gynecological outpatient department was infected.

\section{DISCUSSION}

\section{Importance of Prevention and Control Measures in Gynecological Outpatient Clinics}

After the outpatient temperature screening, medical history inquiry, health code inquiry and temperature screening again, the patients with symptoms were basically eliminated and could not enter the gynecological outpatient department. However, asymptomatic infection could not be completely ruled out. Asymptomatic patients are infectious, and we should try to identify such patients. The hospital environment was relatively enclosed; after entering the inpatient department, there was a high chance of spreading the epidemic. SARS-CoV-2 is highly contagious. The main routes of transmission are respiratory 
The emergency response level for public health emergencies in Hubei Province was adjusted to level two

600

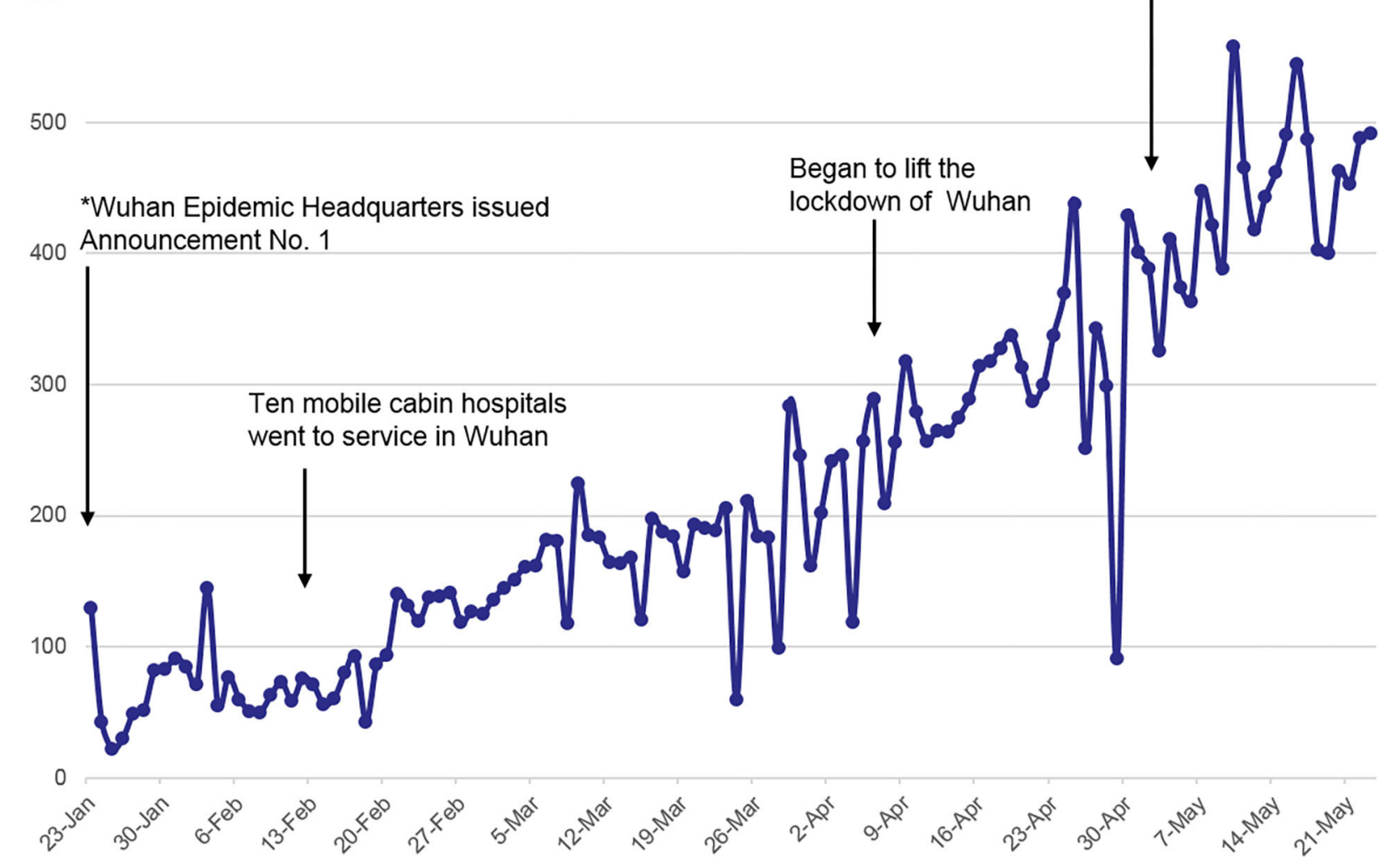

FIGURE 2 | Number of outpatients treated at the Maternal and Child Health Hospital of Hubei province from January 23, 2020, to May 25, 2020. *From 10:00 a.m. on January 23, 2020, the city's urban bus, subway, ferry and long-distance passenger transportation would be suspended, the departure channels of airport and railway stations be temporarily closed. As the condition of the hospital gradually returned to normal, patients traveled smoothly, and the outpatient volume come to recovered.

droplets and close contact transmission. There may also be air transmission and fecal-oral transmission. Although there is no evidence showing the existence of SARS-CoV-2 in the vagina, the patients and doctors shared the same space with limited air flow, and there was a possibility of infection. SARS-CoV-2 nucleic acids can be detected in blood; thus during outpatient procedures, such as family planning surgery, hysteroscopy, and tubal fluid infusion, the healthcare workers may be contaminated by blood. Therefore, outpatient doctors have a certain risk of infection, and outpatient control is very important.

\section{Gynecological Outpatient "Infection Control" Measures During the Epidemic Period}

The goals were to establish treatment and screening procedures for patients during the epidemic, reduce cross infection among patients, family members and healthcare workers, and ensure patients obtain safe and appropriate medical services. Patients were encouraged to seek medical treatment online and open telemedicine channels. Attention was given to the training of healthcare workers, combined with relevant guidelines and consensus recommendations, so that healthcare workers could master the protection procedures and constantly improve the treatment process. Outpatient patients used an appointment system, and patients with appointments made online were permitted to enter the outpatient hall. One patient was permitted per consultation room to reduce the number of people gathered together. In the process of treatment, it was important to pay attention to protective measures for the healthcare workers, disinfect the examination bed immediately, and put biologically contaminated specimens into specimen bags for inspection. Patients who needed to be admitted to the hospital and those who were to undergo outpatient surgery were screened according to the outpatient process.

\section{Changes in Gynecological Outpatient Services During the Epidemic Period}

During the epidemic period, due to the closed management of each community, it was not easy for patients to go out. Therefore, all the patients who came to the hospital were in emergency situations. The main types of problems in the outpatient department were threatened abortion and early pregnancy. There was abnormal uterine bleeding, inevitable abortion, ectopic pregnancy, rupture of the corpus luteum and other emergencies that needed to be treated immediately. The patient's illness, as well as worry about the epidemic situation, made the patient's mood more anxious, so the outpatient healthcare workers should 


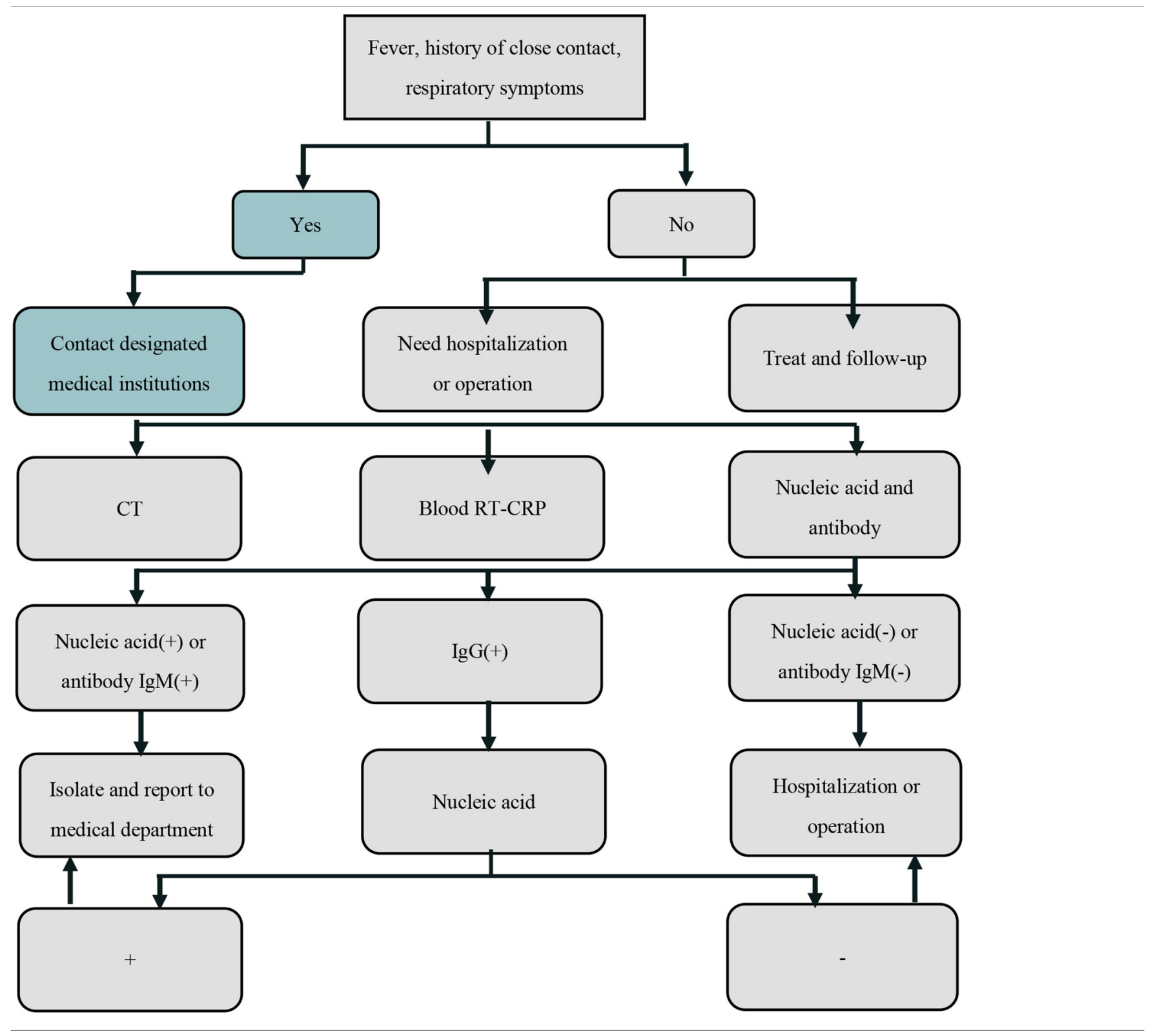

be more patient than usual. Outpatient work became more cumbersome than before. Triage nurses needed to measure body temperature, collect outpatient questionnaires, etc., and the time to process a single patient became significantly longer than before, especially for patients with surgical needs or the need for hospitalization. Patients and their families sometimes needed multiple follow-up visits according to the outpatient screening process, and the waiting time for results was increased. Patients could turn to the emergency department during the waiting period. Therefore, the process should be fully explained before the patient visits so that the patient can understand the process and provide informed consent to avoid disputes caused by repeated examinations.

During the outbreak of SARS-CoV-2, disease prevention and control measures should be standardized as part of the treatment process, which is conducive to the safe continuation of gynecological outpatient work. We have described the working setting of the gynecology outpatient clinic. We hope our experience can help other gynecological colleagues overcome the epidemic as soon as possible.

\section{DATA AVAILABILITY STATEMENT}

The raw data supporting the conclusions of this article will be made available by the authors, without undue reservation.

\section{ETHICS STATEMENT}

The studies involving human participants were reviewed and approved by the Medical ethics committee of the Maternal 
and Child Health Hospital of Hubei Province. Written informed consent for participation was not required for this study in accordance with the national legislation and the institutional requirements.

\section{AUTHOR CONTRIBUTIONS}

LZ and DY conceptualized the study design. DY, JD, and XZ designed the work flow and collected data. DY, KY, and JD plotted the figures and analyzed the data. DY wrote the initial drafts of the manuscript. KY and $\mathrm{XZ}$ revised the manuscript. All authors contributed to the article and approved the submitted version.

\section{REFERENCES}

1. Liu Y, Ning Z, Chen Y, Guo M, Liu YL, Gali NK, et al. Aerodynamic analysis of SARS-CoV-2 in two Wuhan hospitals. Nature. (2020) 582:557-60. doi: 10.1038/s41586-020-2271-3

2. Spinazze A, Cattaneo A, Cavallo DM. COVID-19 outbreak in Italy: protecting worker health and the response of the Italian Industrial Hygienists Association. Ann Work Expo Health. (2020) 64:559-64. doi: 10.1093/annweh/wxaa044

3. Morawska L, Milton DK. It is time to address airborne transmission of COVID-19. Clin Infect Dis. (2020) 71:2311-3. doi: 10.1093/cid/ciaa939

4. Morawska L, Tang JLW, Bahnfleth W, Bluyssen PM, Boerstra A, Buonanno $\mathrm{G}$, et al. How can airborne transmission of COVID-19 indoors be minimised? Environ Int. (2020) 142:105832. doi: 10.1016/j.envint.2020.105832

5. Chen Y, Liu QY, Guo DY. Emerging coronaviruses: genome structure, replication, and pathogenesis. J Med Virol. (2020) 92:418-23. doi: 10.1002/jmv.25681

6. Wang WL, Xu YL, Gao RQ, Lu RJ, Han K, Wu GZ, et al. Detection of SARS-CoV-2 in different types of clinical specimens. J Am Med Assoc. (2020) 323:1843-4. doi: 10.1001/jama.2020.3786

7. Zheng SF, Fan J, Yu F, Feng BH, Lou B, Zou QD, et al. Viral load dynamics and disease severity in patients infected with SARS-CoV-2 in Zhejiang province, China, January-March 2020: retrospective cohort study. Brit Med J. (2020) 369:m1443. doi: 10.1136/bmj.m1443

8. Zietsman M, Phan LT, Jones RM. Potential for occupational exposures to pathogens during bronchoscopy procedures. J Occup Environ Hyg. (2019) 16:707-16. doi: 10.1080/15459624.2019.1649414

9. Jones RM. Relative contributions of transmission routes for COVID-19 among healthcare personnel providing patient care. J Occup Environ Hyg. (2020) 17:408-15. doi: 10.1080/15459624.2020.1784427

10. Phan LT, Sweeney DM, Maita D, Moritz DC, Bleasdale SC, Jones RM, et al. Respiratory viruses in the patient environment. Infect Control Hosp Epidemiol. (2020) 41:259-66. doi: 10.1017/ice.2019.299

11. Liang M, Gao L, Cheng C, Zhou Q, Uy JP, Heiner K, et al. Efficacy of face mask in preventing respiratory virus transmission: a systematic

\section{FUNDING}

This study was supported by the Double first-class construction guide special project funds for the Central Finance Special Project. The research was designed, conducted, analyzed, and interpreted by the authors entirely independently of the funding sources. The researchers confirm their independence from funders and sponsors.

\section{ACKNOWLEDGMENTS}

We are grateful to Beijing Taikang Yicai Foundation for their great support to this work.

review and meta-analysis. Travel Med Infect Dis. (2020) 36:101751. doi: 10.1016/j.tmaid.2020.101751

12. Chu DK, Akl EA, Duda S, Solo K, Yaacoub S, Schunemann HJ, et al. Physical distancing, face masks, and eye protection to prevent person-to-person transmission of SARS-CoV-2 and COVID-19: a systematic review and metaanalysis. Lancet. (2020) 395:1973-87. doi: 10.1016/S0140-6736(20)31142-9

13. Buonanno G, Morawska L, Stabile L. Quantitative assessment of the risk of airborne transmission of SARS-CoV-2 infection: prospective and retrospective applications. Environ Int. (2020) 145:106112. doi: 10.1016/j.envint.2020.106112

14. Buonanno G, Stabile L, Morawska L. Estimation of airborne viral emission: Quanta emission rate of SARS-CoV-2 for infection risk assessment. Environ Int. (2020) 141:105794. doi: 10.1016/j.envint.2020.105794

15. Cherrie JW, Loh M, Aitken RJ. Protecting healthcare workers from inhaled SARS-CoV-2 virus. Occup Med. (2020) 70:335-7. doi: 10.1093/occmed/kqaa077

16. Lin W, Xie Z, Li Y, Li L, Wen C, Cao Y, et al. Association between detectable SARS-COV-2 RNA in anal swabs and disease severity in patients with coronavirus disease 2019. J Med Virol. (2020). doi: 10.1002/jmv.26307. [Epub ahead of print].

17. Peng L, Liu J, Xu W, Luo Q, Chen D, Lei Z, et al. SARS-CoV-2 can be detected in urine, blood, anal swabs, and oropharyngeal swabs specimens. J Med Virol. (2020) 92:1676-80. doi: 10.1002/jmv.25936

Conflict of Interest: The authors declare that the research was conducted in the absence of any commercial or financial relationships that could be construed as a potential conflict of interest.

Copyright (c) 2021 Yao, Yan, Duan, Zhang and Zhou. This is an open-access article distributed under the terms of the Creative Commons Attribution License (CC BY). The use, distribution or reproduction in other forums is permitted, provided the original author(s) and the copyright owner(s) are credited and that the original publication in this journal is cited, in accordance with accepted academic practice. No use, distribution or reproduction is permitted which does not comply with these terms. 